\title{
A LÓGICA DE CLASSES DE BOOLE E A LEI INDEX ${ }^{1}$
}

\begin{abstract}
Alessandro Bandeira Duarte ${ }^{2}$
RESUMO

No presente artigo, discutirei a afirmação de Boole segundo a qual lógica não deve ser associada à metafísica. A visão de Boole depende de uma interpretação particular da Lei Index como uma lei algébrica, da qual o princípio de contradição é derivado. Mostrarei que a visão de Boole é equivocada, uma vez que temos que rejeitar a interpretação algébrica da Lei Index. Além disso, apresentarei o sistema da lógica de classes de Boole, mostrando as vantagens e desvantagens de seu sistema. Apresentarei como derivar Barbara e Celarent a partir dos axiomas e discuto que o método de solução de equações não pode ser considerado um método de dedução.
\end{abstract}

Palavras-chave: Boole. Lei Index. lógica de Classes. Axiomas. Método de Solução de Equações.

\begin{abstract}
In this paper, I will discuss Boole's claim that logic should not be associated with metaphysics. Boole's position depends on a particular interpretation of the Index Law as an algebraic law, from which the principle of contradiction is derived. I will show that Boole's view is misleading since we have to refuse the algebraic interpretation of the Index Law. In addition, I will present Boole's logic of classes, showing the advantages and disadvantages of his system. I will present how to derive Barbara and Celarent from the axioms and discuss that the method for solving equations can not be regarded a method of deduction.
\end{abstract}

Keywords: Boole. Index Law. logic of Classes. Axioms. Method for Solving Equations.

\footnotetext{
${ }^{1}$ Eu gostaria de agradecer à(ao) referee anônima(o) pelas valiosas contribuições ao presente artigo.

${ }^{2}$ Professor do departamento de filosofia da UFRRJ.

E-mail: dedekindbr@nulfic.org. ORCID: 0000-0002-4011-5000.
} 


\section{Introdução}

Em 1847, Boole publicou um pequeno livro intitulado The mathematical analysis of $\operatorname{logic}^{3}$, no qual ele introduziu um sistema algébrico de notação capaz de representar as proposições categóricas que participam dos silogismos. Além disso, esse sistema foi apresentado axiomaticamente, de forma que os silogismos foram derivados de leis do pensamento, que são leis algébricas ${ }^{4}$ 5. Em 1854, The laws of thought foi publicado. Nesse livro Boole melhorou e estendeu as ideias apresentadas em 1847.

Qual é o papel de Boole na história da lógica? Meu objetivo aqui é considerar a análise que Chateaubriand sustentou no artigo "Boole on Reference and Universe of Discourse: Reply to John" ${ }^{6}$. Segundo Chateaubriand, Boole é uma figura importante na história da lógica, não nega que Boole, de fato, pode ser considerado o iniciador da lógica moderna e que lógica algébrica de Boole desempenhou certo papel na lógica desenvolvida no fim do século 19 e início do século 20. Porém, ele considera que os trabalhos em lógica de Frege e Russell são mais essenciais, porque estes últimos consideram a lógica como uma ciência a qual trataria dos aspectos mais fundamentais da realidade.

Para Chateaubriand, o que distingue a noção de lógica de Frege e Russell da noção de lógica de Boole é a concepção metafísica ${ }^{7}$. Aqui temos um ponto interessante ao qual será dada uma maior atenção adiante. Boole defende que a lógica é uma ciência ${ }^{8}$. Ele defende que a lógica trata da relação

\footnotetext{
${ }^{3} \mathrm{O}$ interesse de Boole pela lógica começa com a controvérsia entre William Hamilton e Augustus De Morgan em relação à autoria da teoria da quantificação de predicados. Cf. BOOLE, 1998, p. 1.

${ }^{4}$ Boole dividiu as proposições da lógica em dois tipos: (1) proposições primárias, que estabelecem relações entre classes e (2) proposições secundárias, que estabelecem relações entre proposições. Os axiomas algébricos estabelecidos (seção 3) por Boole valem tanto para proposições primárias como para proposições secundárias.

5 As leis da lógica de Boole são algébricas em um sentido mais estrito. Mais adiante discutirei esse ponto.

${ }^{6}$ Alguns autores consideram o papel de Boole apenas secundário. Em geral, essa interpretação está atrelada à falta de uma teoria da quantificação no sistema de Boole. Veja, por exemplo, Dummett (1967, p. 66) e Heijenoort (1981, p vi). Outros autores defendem o papel proeminente de Boole na história da lógica. Dentre estes, posso mencionar Quine (1940, p. 1), Lewis e Langford (1959, p. 89), Corcoran (2003, p. 273) e Corcoran (2004, p. 163).

${ }^{7}$ Cf. CHATEAUBRIAND, 2004, p. 178-9.

${ }^{8}$ Veja, por exemplo, Boole (1998, p. 7) e Boole (2003, cap. 1).
} 
entre classes ou da relação entre proposições. O próprio Corcoran (2003) afirma que

Boole laid down the groundwork for science of formal laws of being, in Tarski's words 'general laws governing the concepts common to all sciences' or 'the most general laws of thinkables' (1941/1994, pp xii), to use the words that Kneale and Kneale applied to what Boole called laws of thought (1962/ 1988, p. 407). (CORCORAN, 2003, p. 286).

Então, por que Boole afirma que a lógica não deveria mais ser associada à metafísica? A resposta que será sugerida é a seguinte: na sua tentativa de defender que a lógica é uma espécie de álgebra, Boole foi incapaz de ver que seus princípios lógicos, em particular, a Lei Index

$$
x . x=x \text { ou } x^{2}=x
$$

baseava-se em princípios metafísicos e não em princípios algébricos.

Na próxima seção, apresentarei e discutirei os símbolos algébricos primitivos da lógica de Boole e a proposta de tradução das proposições categóricas nessa linguagem. Na terceira seção, discutirei as leis do pensamento (axiomas) do sistema de Boole. Argumentarei que, de acordo com Boole, esses axiomas são verdadeiros quando os símbolos eletivos ${ }^{9}$ recebem os valores 0 ou 1. Em particular, a lei Index só é algebricamente verdadeira nessa interpretação. Em minha visão, esse fato é a razão pela qual Boole rejeita o fundamento metafísico da lógica. Além disso, argumentarei que essa interpretação impede que Boole aceite algumas leis lógicas com respeito às classes, tais como

$$
x+x=x \text { e } 1+x=x
$$

Finalmente, na seção 4, discutirei a derivação de algumas figuras do silogismo aristotélico dentro do sistema de Boole e abordarei alguns problemas relacionados com a classe indeterminada $v$ e com o método de solução de equações.

\footnotetext{
${ }^{9}$ Na próxima seção, explico o que significa "símbolo eletivo".
} 


\section{Os símbolos primitivos e a interpretação algébrica das sentenças categóricas}

\subsection{Os símbolos primitivos}

Não há nos dois livros de Boole já mencionados qualquer resquício do estabelecimento de uma sintaxe formal da linguagem lógica, ou seja, não encontramos nos textos dele um vocabulário inicial, nem regras sintáticas de formação de expressões bem-formadas. Entretanto, é possível reconhecer minimamente qual é o vocabulário inicial e as regras de formação. O vocabulário inicial do sistema de Boole consiste nos seguintes símbolos:

(1) o símbolo “1”;

(2) o símbolo " 0 ";

(3) os símbolos $v, v^{\prime}$, etc;

(4) os símbolos “ $x "$, " $y$ ", " $z$ ", etc;

(5) os símbolos de operadores: “+”, “.”, e “-”

(6) o símbolo de relação: “=”.

De acordo com Boole, símbolo "1" significa a classe universal, que contém todas as classes de indivíduos, se existentes ou não ${ }^{10}{ }^{11}$. Em The mathematical analysis of logic, Boole introduz o símbolo " 0 " sem muitas explicações, todavia o contexto deixa claro que se trata da classe vazia. Os símbolos " $v$ ", " $v$ ", etc podem ter, pelo menos, duas interpretações: ora eles funcionam como classes indefinidas, ora funcionam como espécies de quantificadores que se anexam a uma determinada classe ${ }^{12}$. A introdução dos símbolos " $v ", ~ " v "$ é um dos pontos mais controversos da lógica de Boole ${ }^{13}$. Os ${ }^{10}$ Cf. BOOLE, 1998, p. 15.

${ }^{11} \mathrm{Na}$ verdade, Boole muda sua interpretação de "1" como sendo o universo de discurso fixo (em Mathematical Analysis of Logic) para um universo de discurso variável (em The Laws of Thought). Mas para facilitar esta exposição desconsiderarei este fato.

${ }^{12}$ Boole (1998, p. 39) introduz explicitamente $v$ e $v$ ' para traduzir as sentenças "Alguns Xs são Ys" e "Alguns Ys são Zs". O mesmo procedimento é adotado em Boole (2003, p. 231).

13 Talvez um dos objetivos de Boole ao introduzir a classe indeterminada " $v$ ", como no caso da introdução da classe nula, seria uniformizar a interpretação lógica de sentenças contendo a expressão "Algum X". O que queremos dizer é o seguinte: Boole poderia perfeitamente traduzir "Algum X é Y" por “ $x . y \neq 0$ ”. Contudo, Boole deseja interpretar todas as sentenças como identidades com o objetivo de encontrar um método de dedução. 
símbolos “ $x$ ”, “ $y$ ”, " $z$ ”, etc são símbolos chamados eletivos, uma vez que eles "elegem" do universo (classe universal) um certo subconjunto. Assim, " $x$ " refere-se à classe dos indivíduos do universo que tem a propriedade $\mathrm{X}$, “ $y$ ”, à classe dos indivíduos do universo que tem a propriedade $\mathrm{Y}$ e assim por diante. “+”, “.” e “_” são operadores que agem sobre as classes, produzindo novas classes. “+” funciona como uma espécie de união entre classes; “.”, como uma espécie de interseção; e “-”, como uma espécie de complemento relativo. Entretanto, “+” não representa exatamente a operação de união, pois ela só produzirá um termo, se as classes forem disjuntas. Em relação ao símbolo “-”, há também uma restrição, a saber, a classe no subtraendo tem de ser uma subclasse da classe no minuendo. Finalmente, "=" ("é o mesmo que") representa uma relação entre classes (nos silogismos, trata-se da predicação).

Dados os primitivos lógicos acima, podemos agora afirmar regras sintáticas para produzir expressões bem-formadas na linguagem de Boole. As seguintes regras indutivas sugerem-se:

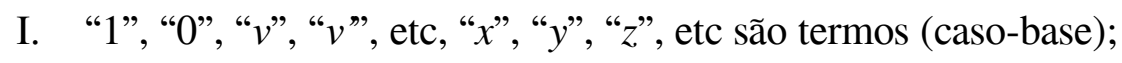

II. se $\mathrm{A}$ e $\mathrm{B}^{14}$ são termos, então A.B é um termo ${ }^{15}$;

III. Se A e B são termos e A.B=0 ${ }^{16}$, então A + B é um termo;

IV. Se A e B são termos e BᄃA, então A - B é um termo;

V. Todo termo é formado a partir das regras I-IV;

VI. Se A e B são termos, então A=B é uma fórmula (equação);

VII.Toda fórmula é formada pela regra VI.

Note que não apresentei nenhuma expressão que denotasse a negação. De fato, Boole não tinha tal expressão. Não obstante, na linguagem de Boole, é completamente possível formar termos negativos. Para isso, Boole utiliza o seguinte artifício: uma vez que "1" denota a classe de todas as coisas, e assu-

\footnotetext{
${ }^{14}$ Aqui “ $A$ " e "B" são metavariáveis que percorrem os termos da linguagem-objeto.

15 Os operadores representam a si mesmo na metalinguagem. Faço isso para evitar a introdução de aspas ou novos símbolos.

16 " 0 " refere-se à classe vazia na metalinguagem. O que pretendo expressar é que as classes devem ser disjuntas para que a união entre elas represente também uma classe.
} 
mindo que " $x$ " denota a classe dos $X \mathrm{~s}$, então " $1-x$ " denotaria a classe das coisas que não são $X \mathrm{~s}$, em outras palavras, " $1-x$ ” pode ser interpretado como o termo negativo "não- $X$ "17. Além disso, é possível mostrar, dada esta interpretação de Boole do termo negativo "não- X", que o par de termos contraditórios exaure o universo. Seja “ $x$ ” a classe dos Xs, seja “1-x" a classe dos não-Xs, então $x+(1-x)^{18}$ é igual a 1 , ou seja, o universo.

\subsection{A interpretação algébrica das sentenças categóricas}

Agora, será analisada a interpretação lógica de Boole das sentenças usadas nos silogismos. Aristóteles, em Prior Analytics, trabalha com quatro tipos de sentenças, a saber:
A. a sentença universal afirmativa (Todo X é Y): $\mathrm{A}(x, y)$;
E. a sentença universal negativa (Nenhum X é Y): $\mathrm{E}(x, y)$;
I. a sentença particular afirmativa (Algum X é Y): I $(x, y)$;
O. e a sentença particular negativa (Algum $\mathrm{X}$ não é $\mathrm{Y})$ : $\mathrm{O}(x, y)^{19}$.

Para Boole, só há um tipo de conector "é mesmo que”, representado pelo símbolo "=". Este fato ocorre porque Boole interpreta "Todo X", "Nenhum X"20, "Algum X" como classes, de forma que "Todo X é Y" significa "a classe inteira $\mathrm{X}$ é $\mathrm{Y}^{\text {”21 }}$. O “é” representa a identidade entre a classe inteira $X$ e uma subclasse da classe $Y$.

Assim, na linguagem simbólica de Boole, as quatro sentenças acima são interpretadas da seguinte forma:

\footnotetext{
${ }^{17}$ Certamente a classe $\mathrm{X}$ é um subconjunto da classe universal, então " $1-x$ ” é um termo.

18 A classe dos Xs e a classe dos não-Xs são disjuntas, assim “ $x+(1-x)$ ” é um termo.

19 Essas abreviações serão usadas mais adiante.

${ }^{20} \mathrm{Na}$ verdade, "Nenhum X" não denota uma classe propriamente dita. Boole transforma a sentença na qual ocorre esta expressão (universal negativa) em uma sentença onde tal ex pressão desaparece. Por exemplo, "Nenhum X é Y" significa a mesma coisa que "Todo X é não-Y” (por obversão). Esta última sentença é aceitável para Boole, porque "Todo X" denota a classe dos Xs e "não-Y" pode ser perfeitamente expresso na sua linguagem. Cf. BOOLE, 2003 , p. 228

${ }^{21}$ Cf. BOOLE, 2003, p. 228.
} 

A'. Todo X é Y: $x \cdot(1-y)=0^{22}$ ou $x=x \cdot y^{2324}$
E'. Nenhum X é Y: $x \cdot y=0^{2526}$
I'. Algum X é Y: $x \cdot y=v^{27}$
O'. Algum X não é Y: $x .(1-y)=v^{28}$

Aqui, temos de fazer algumas observações. A princípio, parece que Boole não assume a quantificação de predicados, mas isto está implicitamente assumido nas fórmulas. Por exemplo, " $x=x . y$ " implica que $x$ é uma subclasse de $y$ e assim a classe dos $x$ é igual a alguma subclasse da classe dos $y$. Portanto, implicitamente, " $Y$ " é entendido em (A') como "Algum $Y$ "29. Caso contrário, (A') afirmaria a extensionalidade entre as classes dos Xs e Ys $(x=y)$. De fato, se ocorrer que $x=x . y$ e $y=y \cdot x$, então $x=y$. A notação acima foi apresentada por Boole (1998). Já em The Laws of Thought, Boole explicitamente apresenta a quantificação de predicados. (A') é expressa lá da seguinte forma:

$$
\text { A”. Todo X é Y: } x=v \cdot y
$$

Na verdade, Boole (2003, p. 228) assume explicitamente, na sua notação, a quantificação do predicado para as sentenças de (A')-(O'). Ele escreve:

The expressions of the eight fundamental types of proposition in the language of symbols are as follows:

$$
\begin{array}{ll}
\text { 1. All Y's are X's } & y=v x \\
\text { 2. No Y's are X's } & y=v(1-x) \\
\text { 3. Some Y's are X's } & v y=v x \\
\text { 4. Some Y's are not-X's } & v y=v(1-x) \\
\text { 5. All not-Y's are X's } & 1-y=v x \\
\text { 6. No not-Y's are X's } & 1-y=v(1-x)
\end{array}
$$

\footnotetext{
${ }^{22}$ A interseção entre a classe dos $X$ s e a classe dos não- $Y$ s é (igual a) a classe nula.

23 A classe dos $X$ s é (igual a) a interseção da classe dos $X \mathrm{~s}$ e da classe dos $Y$ s.

${ }^{24}$ Cf. BOOLE, 1998, p. 21.

25 A interseção da classe dos $X \mathrm{~s}$ e da classe dos $Y_{\mathrm{S}}$ é (igual a) a classe nula.

${ }^{26}$ Cf. BOOLE, 1998, p. 21.

27 A interseção da classe dos $X \mathrm{~s}$ e da classe dos $Y_{\mathrm{S}}$ é (igual a) uma classe indeterminada de Vs que pertencem às classes dos Xs e dos $Y_{\mathrm{s}}$. Cf. BOOLE, 1998, p. 21.

${ }^{28}$ A interseção da classe dos Xs e dos não-Ys é (igual a) uma classe indeterminada de Vs que pertencem às classes dos Xs e dos não-Ys. Cf. BOOLE, 1998, p. 22.

${ }^{29}$ Há passagens que sugerem esta interpretação em The Mathematical Analysis of Logic. Cf. BOOLE, 1998, p. 21. Veja também Boole (1998, p. 24).
} 


$$
\begin{array}{ll}
\text { 7. Some not-Y's are X's } & v(1-y)=v x \\
\text { 8. Some not-Y's are not-X's } & v(1-y)=v(1-x) .
\end{array}
$$

Esta passagem sugere que o termo-predicado " $\mathrm{X}$ " tem de ser entendido por "Algum X"

Boole (1998) estende a concepção das sentenças que podem ser usadas nos silogismos admitindo termos-sujeito negativos. Além disso, ele admite termos complexos (tanto termo-sujeito como termo-predicado). Assim é perfeitamente possível expressar a sentença "Todas as baleias que são azuis são mamíferas" na notação de Boole:

$$
x \cdot y(1-z)=0 \text { ou } x \cdot y=v \cdot z
$$

onde " $x$ " significa a classe das baleias, " $y$ ", a classe das coisas azuis e " $z$ ", a classe dos mamíferos ${ }^{31}$.

\section{As leis do pensamento}

$\mathrm{Na}$ introdução, afirmei que Boole defende a ideia de que a lógica é uma ciência. Neste sentido, ele poderia ser colocado ao lado de Frege, Russell e Gödel. É interessante que um dos pontos do artigo de Chateaubriand (2004) - em resposta ao artigo de Corcoran (2004) - é que ele defende uma concepção metafísica de lógica (lógica como ciência), diferentemente de Boole (e talvez de Corcoran). Aqui, acredito que Chateaubriand foi enganado pelas palavras de Boole quando ele diz que "we ought no longer to associate Logic and Metaphysic" (BOOLE, 1998, p. 13). De fato, Chateaubriand deve ser isentado do engano, porque o próprio Boole não pôde perceber que seus princípios lógicos não eram matemáticos, mas metafísicos. Mas antes de entrar neste assunto, discutirei um pouco mais a visão de Boole de lógica como ciência.

Boole (1998, p. 7) escreve o seguinte:

\footnotetext{
${ }^{30}$ Em seus escritos póstumos a mesma visão é defendida. Cf. BOOLE, 1997, p. 15.

${ }^{31}$ Cf. BOOLE, 2003, p. 35.
} 
It would be premature to speak of the value which this method may possess as an instrument of scientific investigation. I speak here with reference to the theory of reasoning, and to the principle of a true classification of the forms and cases of Logic considered as a Science.

Mas, para Boole, quais são os objetos de estudo da lógica? Chateaubriand defende, em seu livro Logical Forms, que a lógica é uma teoria ontológica de propriedades, em particular, das propriedades lógicas. Para Frege, lógica é uma teoria ontológica de objetos (lógicos) e propriedades. Para Boole, os objetos de estudo da lógica são as classes e suas relações e as proposições e suas relações. Em The Mathematical Analysis of Logic, Boole não expressou explicitamente isso, mas, em The Laws of Thought, ele escreve:

Logic is conversant with two kinds of relations, - relations among things, and relations among fact. But facts are expressed by propositions, the latter species of relation may, at least for the purposes of Logic, be resolved into a relation among propositions. The assertion that the fact or event $\mathrm{A}$ is an invariable consequent of the fact or event B may, to this extent at least, be regarded as equivalent to the assertion, that the truth of the proposition affirming the occurrence of the event $\mathrm{B}$ always implies the truth of proposition affirming the occurrence of the event A. Instead, then, of saying that Logic is conversant with relations among things and relations among facts, we are permitted to say that it is concerned with relations among things and relations among propositions (BOOLE, 2003, p. 7).

É claro, toda ciência tem suas leis. E dentre estas leis, há aquelas que são mais fundamentais (os axiomas), a partir das quais todas as demais são derivadas. Boole está consciente deste fato ${ }^{32}$.

Boole sustenta que é uma função lógica o ato de conceber classes. Além disso, como ciência, a lógica tem de ter suas próprias leis, dentre as quais há as leis mais básicas. Boole propõe o seguinte conjunto de axiomas lógicos (as leis do pensamento):
I. $\mathrm{x}=\mathrm{x}$
II. $\mathrm{x} \cdot \mathrm{y}=\mathrm{y} \cdot \mathrm{x}$
III. $x^{2}=x\left(x^{n}=x\right)^{33}$

\footnotetext{
32 Cf. BOOLE, 2003, p. 5.

${ }^{33}$ De acordo com Boole, a Lei Index é uma lei lógica relacionada às classes, uma vez que o ato de selecionar os Xs da classe universal e novamente selecionar os Xs dessa classe resultante produz novamente a classe dos Xs.
} 


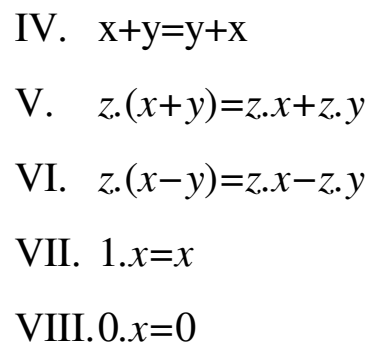

Estas leis são explicita ou implicitamente afirmadas em, pelo menos, um dos dois livros de Boole. (1) é a lei da identidade; (2) afirma que a comutatividade da operação de multiplicação (interseção) de classes; (3) é a Lei Index; (4) afirma a comutatividade da operação de adição (união) de classes; (5) é a lei distributiva da multiplicação em relação à soma (união) de classes; (6) é a lei distributiva da multiplicação em relação à subtração de classes; (7) afirma que a interseção entre a classe universal e qualquer outra classe resulta nesta mesma classe; e (8) afirma que a interseção entre a classe nula e qualquer outra classe resulta na classe nula.

Boole também tem algumas regras de inferências as quais chamarei, seguindo Corcoran (1980):

Regra da funcionalidade da Adição (RFA): $x=y, z=w \vdash x+z=y+w$

Regra da Funcionalidade da Subtração (RFS): $x=y, z=w \vdash x-z=y-w$

Regra da Funcionalidade da Multiplicação (RFM): $x=y, z=w \vdash$ $x . z=y \cdot w$

Casos particulares das regras acima são, respectivamente:

(RFA'): $x=y \vdash x+z=y+z$

(RFS'): $x=y \vdash x-z=y-z$

(RFM'): $x=y \vdash x . z=y . z^{34}$

Segundo Corcoran (1980, p. 616), Boole implicitamente sugeriu como regras a simetria e a transitividade da identidade.

$\overline{{ }^{34} \text { Cf. BOOLE, 2003, p. } 36 .}$ 
(SI): $x=y \vdash y=x$
(TI): $x=y, y=z \vdash x=z$

E também, em uma nota na página 18 de The mathematical analysis of logic, Boole menciona as regras:

$$
\begin{aligned}
& \text { (IgT): } x=z, y=z \quad \vdash x=y \\
& \text { (DiT): } x=z, y \neq z \quad \vdash x \neq y
\end{aligned}
$$

Contudo, (DiT) não tem qualquer utilidade para Boole, uma vez que desigualdades não são expressas em seu sistema ${ }^{35}$.

Há no conjunto de axiomas de Boole algumas omissões de leis que poderiam ser consideradas axiomas lógicos. Em primeiro lugar, Boole não assume explicitamente nem a associatividade da multiplicação, nem a associatividade da adição de classes. De acordo com Corcoran (1980, p. 616), “...perhaps it was his casual attitude concerning parentheses which led him [Boole] to overlook the two associative laws".

Contudo, há outras leis que não foram afirmadas por Boole, porque, acredito, elas iriam de encontro com a sua ideia de que a lógica é uma espécie de matemática (uma álgebra da mente). Em uma passagem citada acima, Boole afirma que a lógica tem de ser associada à matemática e não mais à metafísica. Essa afirmação é sustentada pelo seguinte fato, a saber, da Lei Index, Boole deriva uma fórmula que pode ser interpretada como o Princípio de Contradição. Ele escreve:

That axiom of metaphysicians which is termed the principle of contradiction, and which affirms that it is impossible for any being to possess a quality, and at the same time not possess it, is a consequence of the fundamental law of thought, whose expression is $x^{2}=x$.

Let us write this equation in the form

$$
x-x^{2}=0
$$

\footnotetext{
35 Boole não tem símbolo para negação de fórmulas. Além disso, “ $\neq$ ” não é primitivo no sistema.
} 
whence we have

$$
x \cdot(1-x)=0
$$

both these transformations being justified by the axiomatic laws of combination and transposition (II.13) (BOOLE, 2003, p. 49) ${ }^{36}$.

Ora, em que sentido Boole acredita que a lógica não depende mais da metafísica? Para Boole, os axiomas lógicos apresentados acima são axiomas algébricos $^{37}$. Em um artigo póstumo, Boole escreve o seguinte:

[1st] It has been shewn that if $x$ and $y$ are any two elective symbols then

$$
x+y=y+x .
$$

Now the same equation holds true if $x$ and $y$ represent numbers. We know $7+5=5+7,6+2=2+6$ and generally whatever numerical values we attach to $x$ and $y$ that $x+y=y+x$.

2nd. It has also been shewn that $x$ and $y$ being any elective symbols we have

$$
\mathrm{x} \cdot \mathrm{y}=\mathrm{y} \cdot \mathrm{x}
$$

and this relation is equally true if $x$ and $y$ are any arithmetical symbols. Thus $7 \times 5=5 \times 7,8 \times 9=9 \times 8$ and similarly for any other values of $x$ and $y \ldots$

4th. Any elective symbol $x$ satisfies the law whose expression is

$$
x^{2}=x
$$

It is not true that any numerical symbol satisfies this law. Out of the entire range of numerical magnitude from minus infinite to plus infinity there are but two magnitudes which satisfies this law they are 0 and 1 . For we have

$$
0 \times 0=0 \text { or } 0^{2}=0
$$

and

$$
1 \times 1=1 \text { or } 1^{2}=1 .
$$

These numerical magnitudes may in fact be introduced into the elective system and admit of interpretation there as well as in the system of number to which they also belong (BOOLE, 1997, p. 7 $8)$.

\footnotetext{
${ }^{36}$ Embora Boole não tenha mencionado este fato em The Mathematical Analysis of Logic, pode ser que ele já tivesse isso em mente.

${ }^{37}$ Cf. BOOLE, 2003, p. 50.
} 
De acordo com essa passagem, podemos dividir os axiomas em duas classes, a saber, aqueles que são válidos para quaisquer valores numéricos e aquele que é válido para valores numéricos restritos. Formam a primeira classe os axiomas 1), 2), 4), 5), 6), 7) e 8). A segunda classe é formada justamente pelo axioma 3), a Lei Index, cuja validade se dá para valores 0 ou 1: $0^{2}=0$ $\left(0^{n}=0\right)$ e $1^{2}=1\left(1^{n}=1\right)$.

Em Laws of Thought, Boole também defende que a sua álgebra de classe é uma álgebra que pode ser interpretada, tomando-se os valores 0 e 1 para os símbolos eletivos ${ }^{38}$. É nesse sentido que sua Lei Index é uma lei algébrica e, portanto, que a Lei de Contradição é uma lei algébrica (derivada) e não um axioma metafísico.

Agora, podemos entender porque no sistema de lógica de Boole, não há os axiomas

$$
\begin{aligned}
& \text { 1) } x+x=x \text { ou } 2 \cdot x=x \\
& \text { 2) } 1+x=x^{39}
\end{aligned}
$$

Suponha que " $x$ " tome os valores 0 ou 1 . As leis acima serão verdadeiras somente se " $x$ " for igual a $0^{40}$. Portanto, parece-nos, é totalmente justificado, dada a interpretação de Boole, a não-inclusão destas leis. Para mim, elas não foram simples omissões. Aceitá-las significaria dizer que não havia nenhuma analogia entre os axiomas lógicos e as leis algébricas e, portanto, a redução da lógica à matemática estaria fadada ao fracasso.

É interessante mencionar que, para Boole, a Lei Index não funciona quando aplicada a uma adição de classes. Por exemplo, a equação$$
(x+y)^{2}=x+y
$$
que se poderia adicionar essas leis. Como tentei argumentar, isso não é possível para Boole. Corcoran também afirma que não há em The mathematical analysis of logic a lei de não-contradição. Ora, essa lei é uma consequência direta da Lei Index. Portanto, para mim, não se trata exatamente de uma omissão. Corcoran ainda menciona a omissão das leis de De Morgan. Aqui, acredito, essas leis também não poderiam ser válidas na interpretação de Boole, pois " $x+y$ ” só produzirá um termo, se as classes forem disjuntas.

${ }^{40}$ Na verdade, sintaticamente “ $x+x$ ” não pode ser termo. Logo, “ $x+x=x$ ” não é fórmula.
} 
Perspectiva Filosófica, vol. 47, n. 2, 2020

não é válida no sistema de Boole. Pois, fatorando, $(x+y)^{2}$ é igual a

$$
\begin{aligned}
& x^{2}+2 \cdot x \cdot y+y^{2} . \\
& \text { Como } x^{2}=x \text { e } y^{2}=y, \text { temos } \\
& x+2 \cdot x \cdot y+y .
\end{aligned}
$$

Todavia, "2.x.y” não pode ser eliminado na interpretação lógica no sistema de Boole.

Porém, se assumirmos (1) e (2), a igualdade vale. Por (1), temos que

$x+x \cdot y+y$.

Ora, daí é obtido que

$x \cdot(1+y)+y$.

E por meio da fórmula (2), temos

$x .1+y$.

Mas pelo axioma (7), temos então

$x+y$.

A Lei Index vale para termos complexos produzidos por meio da multiplicação de classes. Assim, em geral, o seguinte vale:

3) $(x \cdot y)^{2}=x \cdot y$ 
De $(x \cdot y)^{2}$, obtemos $x^{2} \cdot y^{2}$. Mas $x^{2}=x$ e $y^{2}=y$. Logo, $x \cdot y$.

A Lei Index também vale para o seguinte termo: $1-x$.

4) $(1-x)^{2}=1-x$

De $(1-x)^{2}$, obtemos $1^{2}-2 \cdot x+x^{2}$. Mas $1^{2}=1$ e $x^{2}=x$. Assim, temos $1-2 . x+x$. Ou seja, $1-x^{41}$.

De acordo com a minha interpretação dada nessa seção, Boole confundiu-se. A Lei Index e o Princípio de Contradição são equivalentes. Na sua ânsia de eliminar a interpretação metafísica do último, Boole direcionou sua álgebra da lógica para um caminho tortuoso. Por isso, ele não pôde expressar leis lógicas que são bastante naturais. De fato, os sucessores de Boole logo assumiram (1) e (2).

\section{O tratamento de Boole dos silogismos}

Um dos objetivos de Boole em The Mathematical Analysis of Logic (e também em The laws of thought) é deduzir, dentro da sua linguagem lógica, os silogismos até então aceitos, bem como mostrar a invalidade de certos argumentos. Vale mencionar o que é um silogismo. De acordo com Aristóteles, um silogismo é formado por um conjunto de proposições a partir do qual uma outra proposição (a conclusão) necessariamente se segue.

Aristóteles não impõe nenhuma restrição no número de proposições utilizadas em um silogismo. Não obstante, em Prior Analytics, Aristóteles menciona apenas argumentos compostos de três proposições, sendo duas premissas e uma conclusão. As deduções de Aristóteles dos silogismos são executadas usando-se oito regras, as quais três são regras de conversão, quatro são silogismos perfeitos e uma é a regra de redução ao absurdo ${ }^{42}$. As regras de conversão são: regras de conversão total (ou simples), regra de conversão parcial.

\footnotetext{
41 Também vale para o termo " $\mathrm{x}+(1-\mathrm{x})$ ": $[\mathrm{x}+(1-\mathrm{x})]^{2}=\mathrm{x}+(1-\mathrm{x})$.

42 A prova por redução ao absurdo é necessária, quando uma das premissas é particular negativa. Aristóteles também usa ecthesis, mas não entraremos em detalhes.
} 
Regra de Conversão Simples A (RCT A): E $(x, y) \vdash \mathrm{E}(y, x)$
Regra de Conversão Simples B (RCT B): $\mathrm{I}(x, y) \vdash \mathrm{I}(y, x)$
Regra de Conversão Parcial (RCP): A $(x, y) \vdash \mathrm{I}(y, x)$

Os silogismos perfeitos são os silogismos da primeira figura ${ }^{43}$ que são:

Barbara: $\mathrm{A}(x, y), \mathrm{A}(y, z) \vdash \mathrm{A}(x, z)$

Celarent: $\mathrm{A}(x, y), \mathrm{E}(y, z) \vdash \mathrm{E}(x, z)$

Darii: $\mathrm{I}(x, y), \mathrm{A}(y, z) \vdash \mathrm{I}(x, z)$

Ferio: $\mathrm{I}(x, y), \mathrm{E}(y, z) \vdash \mathrm{O}(x, z)$

Agora, há elementos suficientes para deduzir outros silogismos. Por exemplo, é possível deduzir o seguinte silogismo da segunda figura:

Cesare: $\mathrm{E}(x, y), \mathrm{A}(z, y) \vdash \mathrm{E}(z, x)$

Para provar isso, basta aplicar (RCT A) em E(x,y). Então é obtido $\mathrm{E}(y, x)$. De $\mathrm{E}(y, x)$ e $\mathrm{A}(z, y)$, de acordo com Celarent, é obtido $\mathrm{E}(z, x)$.

É importante também mencionar a maneira pela qual Aristóteles exclui certos argumentos como silogísticos. Aristóteles afirma, como regra geral para a segunda figura do silogismo, que uma das premissas tem de ser negativa. Se ambas as premissas forem afirmativas, nenhuma conclusão seguir-se-á por necessidade. Por exemplo, assuma o seguinte argumento como silogístico:

$$
\mathrm{A}(x, y), \mathrm{A}(z, y) \vdash \mathrm{A}(z, x)
$$

É possível mostrar que as premissas podem ser verdadeiras e que a conclusão pode ser falsa. Suponha que " $x$ ", “ $y$ ” e " $z$ ” sejam, respectivamente, "homem", mamífero" e "baleia". Neste caso, as premissas são verdadeiras,

\footnotetext{
${ }^{43}$ A divisão das figuras do silogismo em primeira, segunda, terceira e quarta tem a ver com a posição do termo médio, o termo que é eliminado da conclusão. Na primeira figura, o ter mo médio ora termo-sujeito, ora termo-predicado. Na segunda figura, o termo médio aparece como termo-predicado nas duas premissas; e na terceira figura, o termo médio aparece como termo-sujeito nas duas premissas. A quarta figura é parecida com a primeira, mas os termos na conclusão são trocados.
} 
mas a conclusão é falsa. É desta forma que Aristóteles mostra a invalidade de $\operatorname{argumentos}{ }^{44}$.

Dada esta pequena exposição do sistema de dedução de Aristóteles, voltemo-nos a Boole. Um dos pontos de distinção do sistema de dedução de Boole em relação ao de Aristóteles é o uso de axiomas lógicos. De acordo com Corcoran (1980, p. 615), "this is a striking qualitative improvement on Aristotle's direct deductions which were constructed using eight rules...but no logical axioms”. Agora, entenderemos por que Boole expressa todas as suas sentenças na forma de uma identidade. Como observado acima, ocorre, nos silogismos, um termo (chamado termo médio) que desaparece na conclusão. Boole tem de ter alguma forma de excluir tal termo no seu sistema de dedução. Ora, algebricamente, há uma maneira de se fazer isto. Por exemplo, sejam as equações

$$
\begin{aligned}
& \text { 1. } 4 x+3 y+2 z=0 \\
& \text { 2. } x+2 y+3 z=0
\end{aligned}
$$

Se quisermos excluir $x$ do sistema, basta multiplicarmos a segunda equação por -4 . Assim, obtemos

$$
\text { 2'. }-4 x-8 y-12 z=0 \text {. }
$$

E somamos a primeira equação por esta última, resultando em

$$
\text { 3. }-5 y-10 z=0 \text { ou } 5 y+10 z=0 \text {. }
$$

Vale mencionar que, nesta derivação, outros passos implicitamente foram dados. Em particular, é assumido que

$$
-4=-4 \text { (identidade) }
$$

\footnotetext{
${ }^{44} \mathrm{Na}$ verdade, o método de Aristóteles é mais completo, refutando qualquer proposição categórica possível como consequência lógica dessas premissas. Mas não entrarei em detalhes.
} 
Também é assumida a regra RFM', tomando-se para “ $x$ ” a expressão “ $x+2 y+3 z "$ e para " $y$ ", " 0 ".

Boole apresenta o seguinte método geral de eliminação de um termo (análogo ao exemplo acima):

\section{Eliminação $1(\text { E1 })^{45}$ :}

$$
\begin{aligned}
& a \cdot y+b=0 \\
& a^{\prime} \cdot y+b=0 \\
& a \cdot b^{\prime}-a^{\prime} \cdot b=0
\end{aligned}
$$

Entretanto, dada a solução de equações, esse esquema de eliminação de termos pode ser substituído, por uma questão de conveniência e praticidade, pelo seguinte outro esquema:

\section{Eliminação $2(\mathbf{E 2})^{46}$}

$$
\begin{aligned}
& b=a \cdot y \\
& a^{\prime} \cdot y=b^{\prime} \\
& a^{\prime} \cdot b=a \cdot b^{\prime}
\end{aligned}
$$

De acordo com Corcoran (1980), tanto E1 como E2 são válidas no sistema lógico de Boole. Seguindo-o, apresentarei a dedução de $\mathbf{E 2}^{47}$ :
1. $b=a \cdot y$
premissa
2. $a^{\prime} \cdot y=b^{\prime}$
premissa
3. $a^{\prime}=a^{\prime}$
Identidade
4. $a^{\prime} \cdot b=a^{\prime} \cdot(a \cdot y)$
1,3 RFM
5. $a=a$
Identidade
6. $a \cdot\left(a^{\prime} \cdot y\right)=a \cdot b^{\prime}$
2,5 RFM
7. $a^{\prime} \cdot(a \cdot y)=\left(a^{\prime} \cdot a\right) \cdot y$
Associatividade da Multiplicação
8. $a \cdot\left(a^{\prime} \cdot y\right)=\left(a \cdot a^{\prime}\right) \cdot y$
Associatividade da Multiplicação
9.(a'a).y=a'.(a.y)
7 , simetria da identidade

\footnotetext{
${ }^{45}$ Veja Boole (1998, p. 32).

${ }^{46}$ Veja Boole (1998, p. 34-5).

47 A derivação será um pouco mais completa em relação à de Corcoran.
} 

10. $a^{\prime} \cdot b=\left(a^{\prime} \cdot a\right) \cdot y$
$9,4, \mathbf{I g T}$
11. $\left(a \cdot a^{\prime}\right) \cdot y=a b^{\prime}$
8,6 IgT
12. $a \cdot a^{\prime}=a^{\prime} \cdot a$
Comutatividade da Multiplicação
13. $y=y$
Identidade
14. $\left(a \cdot a^{\prime}\right) \cdot y=\left(a^{\prime} \cdot a\right) \cdot y$
$12,13, \mathbf{R F M}$
15. $a^{\prime} \cdot b=\left(a \cdot a^{\prime}\right) \cdot y$
14,10, IgT
16. $a \cdot b^{\prime}=\left(a \cdot a^{\prime}\right) \cdot y$
11, Simetria da Identidade
17. $a \cdot b^{\prime}=a^{\prime} \cdot b$
16,15, IgT (QED)

Dado este sistema de eliminação (E2), Boole deduz algumas figuras dos silogismos. Por exemplo, o modo Barbara é derivado da seguinte forma:

$$
\begin{array}{ll}
\text { Todo X é Y A }(x, y) & x .(1-y)=0 \text { ou } x=x . y \\
\text { Todo Y é Z A }(y, z) & y \cdot(1-z)=0 \text { ou } y=y . z
\end{array}
$$

Em primeiro lugar, podemos resolver ambas as equações, obtendo-se: $x=v . y$ e $y=v z^{48}{ }^{49}$. Agora, podemos aplicar E2, ou seja, multiplicando as equações " $x=v \cdot y$ " e " $y=v \cdot z$ " e eliminando " $y$ ", obtemos

$$
x=v \cdot z
$$

que expressa a sentença “Todo X é Z” $(\mathrm{A}(x, z))$.

É interessante mencionar que Boole assume os axiomas lógicos, mas na dedução dos silogismos, ele só usa E2 (ou E1). Porém, como pode ser visto na dedução de E2, muitos axiomas lógicos são usados. De fato, é possível dar uma dedução completa de Barbara usando, em vez de E2, os axiomas lógicos:
1. $x=x \cdot y$
Premissa
2. $y=y \cdot z$
Premissa
3. $z=z$
Axioma 1
4. $z \cdot x=z \cdot(x \cdot y)$
1, 3 RMF
5. $z \cdot(x . y)=(z \cdot y) \cdot x$
4, Associatividade

\footnotetext{
${ }^{48}$ Como já mencionado, essa já seria a interpretação padrão em Boole (2003).

${ }^{49}$ Mais adiante, discuto a solução de equações.
} 

6. $z \cdot y=(z \cdot y) \cdot x$
4,5, IgT
7. $x=x$
Axioma 1
8. $y \cdot x=(y \cdot z) \cdot x$
7,8 RMF
9. $y \cdot x=(z \cdot y) \cdot x$
8 , Comutatividade
10. $z \cdot x=y \cdot x$
6, 9, IgT
11. $z \cdot x=x \cdot y$
10, Comutatividade
12. $z x=x$
1, 11, IgT
13. $x=x . z$
12, Comutatividade

O modo Celarent é derivado da seguinte forma, usando-se E2:
1. Todo X é Y A $(x, y)$
$x=x \cdot y$
Premissa
2. Nenhum Y é Z E $(y, z)$
$y \cdot z=0$
Premissa
3. Nenhum X é Z E $(x, z)$
$x . z=0$
$1,2 \mathbf{E 2}$

A derivação completa de Celarent sem usar E2 pode ser executada da seguinte forma:
1. $x=x \cdot y$
Premissa
2. $y \cdot z=0$
Premissa
3. $x=x$
Axioma 1
4. $x \cdot(y \cdot z)=0$
2,3, RMF
5. $z=z$
Axioma 1
6. $z . x=z .(x \cdot y)$
1, 5, RMF
7. $x . z=x \cdot(y . z)$
6, Comutatividade e Associatividade
8. $x . z=0$
4, 7, Transitividade

De acordo com Corcoran (1980), há três problemas no sistema de dedução de Boole. Em primeiro lugar, diferentemente de Aristóteles, Boole não tem nenhum tipo de dedução indireta (via redução ao absurdo). Segundo Corcoran, uma possível justificativa para isso é a falta de uma expressão que funciona como negação (no seu sentido próprio). Esse é um ponto interessante, mas não entrarei nos detalhes. O segundo problema é o que Corcoran (1980, 2003) chama de "falácia das soluções" (fallacy of solutions). Esta falácia está intimamente ligada ao símbolo " $v$ " que representa a classe indeterminada. 
Boole mostra que as conversões (de sentenças) apresentadas por Aristóteles são deriváveis em seu sistema. A conversão simples é obtida, usando-se o axioma da comutatividade da multiplicação. Por exemplo ${ }^{50}$ :

\section{De "Nenhum X é Y"}

$x \cdot y=0$,

obtemos

y. $x=0$;

de "Algum X é Y"

$\mathrm{x} . \mathrm{y}=\mathrm{v}$

obtemos

$\mathrm{y} \cdot \mathrm{x}=\mathrm{v}$

isto é, “Algum Y é X”.

O problema encontra-se na conversão parcial. Boole escreve:

The equations $\mathrm{A}$ and $\mathrm{E}$, written in the forms

$$
\begin{aligned}
& (1-y)=0 \\
& y \cdot x=0
\end{aligned}
$$

give on solution the respective forms

$$
\begin{aligned}
& \mathrm{x}=\mathrm{vy} \\
& x=v(1-y)
\end{aligned}
$$

the correctness of which may be shewn by substituting these values of $\mathrm{x}$ in the equations to which they belong, and observing that those equations are satisfied quite independently of the nature of the symbol $v "$ (BOOLE, 1998, p. 27).

Para entendermos esta passagem, é necessário entrar na questão "das propriedades das funções eletivas" pios do raciocínio simbólico" (BOOLE, 2003, p. 66-79).

Partindo do teorema Maclaurin ${ }^{52}$, Boole chega ao seguinte resultado: qualquer que seja a função de um símbolo eletivo, temos que

\footnotetext{
${ }^{50}$ Veja Boole (1998, p. 26).

${ }^{51}$ Uma função eletiva é uma função em termos dos símbolos eletivos: $x, y$, $z$, etc.

${ }^{52}$ Este teorema é: $\phi(x)=\phi(0)+\phi^{\prime}(0) x+\frac{\phi^{\prime \prime}(0)}{1.2} x^{2}+\ldots$
} 
Perspectiva Filosófica, vol. 47, n. 2, 2020

$$
f(x)=f(1) x+f(0)(1-x)
$$

Este resultado é mais facilmente obtido em The laws of thought. Assuma uma função qualquer de um símbolo eletivo

$$
f(x)=a x+b(1-x)^{53}
$$

Se $x$ for 0 , temos

$$
f(0)=b \text {. }
$$

Se $x$ for 1 , temos

$$
f(1)=a \text {. }
$$

Substituindo na função original " $a$ " por " $f(1)$ " e " $b$ " por " $f(0)$ ", obte$\operatorname{mos}$

$$
f(x)=f(1) x+f(0)(1-x)(\text { teorema T1) }
$$

Mas qual é importância deste teorema? Ora, podemos considerar a fórmula

$$
x .(1-y)=0(\text { Todo } \mathrm{X} \text { é Y })
$$

da seguinte forma:

$$
x=\frac{0}{1-y} .
$$

${ }^{53}$ Esta expressão é obtida via definição. Cf. BOOLE, 2003, p. 72. 
E podemos expandir, usando T1, a expressão “ $\frac{0}{1-y}$ ” (considerada como uma função de $y$ ). Assim, para $y=1$, temos

$$
\frac{0}{0} y
$$

para $y=0$, temos

$$
\frac{0}{1}(1-y)
$$

Na interpretação de Boole, “ $\frac{0}{1}$ ” representa a classe vazia. E pelo axioma $8,0 .(1-y)=0$. Seguindo a interpretação aritmética que afirma que a divisão de zero por zero é indeterminada, Boole considera que “ $\frac{0}{0}$ ” representa uma classe indeterminada - a classe $v$. Assim, de

$$
x .(1-y)=0 \text {, }
$$

Boole deriva a equação

$$
x=\frac{0}{0} y_{\text {ou }} x=v \cdot y \text {. }
$$

De acordo com Corcoran (1980), o erro de Boole reside no fato dele acreditar que um método de obtenção de solução para uma determinada função é o mesmo que uma dedução.

Vale salientar um problema que é observado e que tem relação com isso. Como disse anteriormente, Boole assume em The laws of thought que a fórmula que expressa a sentença "Todo X é Y" é

$$
x=v \cdot y .
$$


Nesta fórmula, Boole explicitamente afirma a quantificação do predicado. Boole (2003, p. 229) mostra que a fórmula acima implica a fórmula que ele usou em The mathematical analysis of logic, a saber,

$x=x \cdot y$ ou $x \cdot(1-y)=0$.

Para isto, basta multiplicar " $x=v \cdot y$ " por " $1-y$ ":

$x(1-y)=v \cdot y(1-y)^{54}$

Ora, à direita da identidade, obtemos

$v \cdot y-v y^{2}$

Mas, pela Lei Index, temos

$$
\text { v. } y-v y^{55} \text {. }
$$

Deste modo, somos levado à seguinte conclusão: se de $x$. $(1-y)=0$, obtemos $x=v \cdot y$ (assumindo que isto ocorre por meio de uma dedução lógica); e se de $x=v \cdot y$, obtemos $x \cdot(1-y)=0$ (por dedução lógica), então ambas as fórmulas deveriam ser equivalentes. Isso significa o seguinte: todas as conclusões que são derivadas de uma são derivadas da outra e vice-versa. Entretanto, esta afirmação é incorreta! Por exemplo, Boole (1998, p. 35) deriva a conclusão do silogismo da quarta figura (Fesapo) usando a fórmula

$$
y=v x \text {. }
$$

\footnotetext{
${ }^{54}$ Aqui, alguns passos onde são utilizados axiomas lógicos e regras de inferência foram omitidos.

${ }^{55}$ Do axioma 1, temos: $x=x$. Assim, podemos obter o seguinte: $x-x=0$.
} 
A derivação ocorre da seguinte forma:

$$
\begin{array}{ll}
\text { Nenhum Z é Y } & 0=z y \\
\text { Todo Y é X } & y=v x
\end{array}
$$

Usando E2, obtemos

$0=z v x^{56}$

que significa que alguns Xs não são Zs. Aqui vale mencionar a segunda interpretação do símbolo " $v$ ". " $v$ " ocorre, nesta dedução, anexado ao símbolo " $x$ ", de maneira que não poderíamos entender por esta fórmula que "Alguns $\mathrm{Zs}$ não são Xs". " $v$ " parece funcionar como uma espécie de quantificador ${ }^{57}$.

Contudo, se, em vez de $y=v x$, usarmos a fórmula

$$
y(1-x)=0
$$

obtemos, usando E2, o seguinte

$$
0=0^{58} 59 .
$$

Esta equação, como será observado abaixo, é usada por Boole para mostrar a invalidade de um argumento. Portanto, estas duas fórmulas não podem ser equivalentes. Isto significa que o método de conversão parcial de Boole não é uma dedução lógica.

Antes de passarmos para o terceiro e último problema, gostaria de fazer algumas especulações. Se Boole assumisse que a sentença “Todo X é Y” é expressa apenas pela fórmula " $x=v \cdot y$ ” e rejeitasse seu método de conversão parcial, o sistema seria interessante? Por um lado, penso que sim! Como foi visto, a partir desta fórmula, podemos derivar a fórmula " $x(1-y)=0$ ” ou

\footnotetext{
${ }^{56}$ Para aplicar E2, considere: $b=0 ; a=z ; a^{\prime}=1$; e $b^{\prime}=v \cdot x$.

${ }^{57}$ Cf. BOOLE, 1998, p. 37.

58 É só multiplicar as fórmulas " $y(1-x)=0$ " e " $0=z y$ ", eliminando o termo " $y$ ".

${ }^{59}$ Boole não pode usar também $y=y . x$, pois não seria possível eliminar $y$, usando-se E2.
} 
" $x=x . y "$. Além disso, seria possível provar deduções do tipo: "Todo X é Y" implica “Algum X é Y”.
1. $x=v \cdot y$
Premissa
2. $v=v$
Identidade
3. $v \cdot x=v^{2} y$
1,2 RFM
4. $v^{2}=v$
Lei Index
5. $v x=v y$
3,4

A interpretação de (5), segundo Boole, em The Laws of Thought, é "Algum X é (algum) Y"60.

O terceiro problema apontado por Corcoran diz respeito ao critério de invalidade sustentado por Boole. Como escrevi anteriormente, a fim de mostrar a invalidade de um argumento, Aristóteles apresenta um contraexemplo. Boole parece não ter notado a simplicidade e naturalidade deste método. Seu critério para estabelecer a invalidade de um argumento é extremamente complexo e não-natural. Assumamos o exemplo de Boole (1998, p. 35):

De

$x y=x($ Todo $\mathrm{X}$ é $\mathrm{Y})$

e

$z=z y($ Todo $\mathrm{Z}$ é Y $)$

\footnotetext{
${ }^{60}$ A classe indeterminada foi alvo de muitos debates e que seu uso gera incongruências no sistema de Boole. Por exemplo, Peirce (1989, p. 171) mostrou que da fórmula " $v . x=v .(1-$ y)” (Alguns Xs são não-Y) é possível derivar a fórmula “v. $y=v \cdot(1-x)$ )" (Alguns Ys são não$\mathrm{Xs)}$, o que é falacioso (de algum mamífero não é humano não se segue que algum humano não é mamífero). A derivação seria da seguinte forma: assuma a fórmula " $v x=v(1-y)$ ". Ora, multiplicando o segundo termo da equação, obtemos$$
v \cdot x=v-v \cdot y
$$

passando " $v x$ " para direita e " $v y$ " para esquerda (da identidade), obtemos $v \cdot y=v-v \cdot x$

daí obtemos

$$
v \cdot y=v \cdot(1-x)
$$

ou seja, "Alguns Y são não-Xs". Contudo, essa falácia é barrada no sistema de Boole, uma vez que ele assume um conjunto enumerável (?) de símbolos: $v, v$, v", etc. Já mencionamos isso na seção 2. De fato, Boole (2003, p. 231) assume um procedimento semelhante na derivação de Barbara. Se assumirmos esse conjunto enumerável (?), então "algum X não é $\mathrm{Y}$ " poderia ser traduzida por $v \cdot x=v$ '. $(1-y)$ e "algum Y não é X", por $v$ ". $y=v^{\prime \prime} .(1-x)$.
} 
é obtido, usando-se E2,

$x z=x z$

para Boole, isto é o mesmo que

$0=0$.

Segundo Boole, todo argumento que produz esta equação é inválido.Porém, esta não é a única maneira de se mostrar a invalidade de um argumento. Em The Mathematical Analysis of Logic, Boole assume certas equações auxiliares para mostrar a dedução de " $0=0$ ” das premissas. Ele escreve:

Of cases in which no inference is possible, we take as examples AO, Fig. 1, and by mutation, OA, Fig. 4,

$$
\begin{array}{lll}
\text { All Ys are Xs } & y(1-x)=0, & y(1-x)=0 \\
\text { Some Zs are not Ys } & v z=v(1-y) & (a) \quad \begin{array}{c}
v(1-z)=v y \\
\end{array} \\
\cline { 2 - 3 } & & \\
& & 0=0
\end{array}
$$

since the auxiliary equations in this case is $v(1-z)=0$ (BOOLE, 1998, p. 38).

De acordo com Corcoran, estas equações auxiliares estão diretamente ligadas com a interpretação de " $v$ " como uma espécie de quantificador. Se, nos contextos nos quais " $v$ " ocorre, ele significar "Alguns Xs", então a equação auxiliar será " $v(1-x)=0$ ” . Se ele significar "Alguns não-Xs" a equação auxiliar será " $v x=0$ ”. No caso acima, " $v z$ ", em " $v z=v(1-y)$ ", significa "Alguns Zs", daí a necessidade da equação auxiliar " $v(1-z)=0$ ”, para se obter a equação " $0=0$ ”.

${ }^{61}$ Embora confuso, isto tem alguma coerência. Se " $v$ " no contexto " $v x$ " significar "Algum $\mathrm{X}$ ", então a classe " $v$ " é composta apenas de elementos que pertencem à classe $x$. Logo, a interseção entre a classe $v$ e a classe não- $x$ tem de ser vazia. 


\section{Conclusão}

George Boole, ao publicar o livro The mathematical analysis of logic, estabeleceu o primeiro estágio da evolução da lógica matemática moderna. Podemos listar alguns de seus feitos: (1) criação de uma linguagem algébrica na qual é possível (2) expressar leis lógicas, (3) métodos de inferência que preservam verdade (excetuando-se seu método de solução de equações). Além disso, (4) Boole foi capaz de interpretar as sentenças categóricas da silogística aristotélica na sua linguagem e (5) derivar as figuras silogísticas a partir dos axiomas lógicos.

$\mathrm{Na}$ introdução, mencionei a posição defendida por Chateaubriand em relação a Boole, segunda a qual o lógico britânico não pode ser considerado o pai da lógica matemática moderna, pois essa nasceu a partir da concepção metafísica de lógica de Frege e Russell (lógica como ciência). De fato, tanto em The mathematical analysis of logic como em The laws of thought Boole sustentou que os princípios lógicos não são metafísicos, mas matemáticos. Entretanto, como tentei mostrar na seção 3, essa posição de Boole trata-se de um erro, que levou seu sistema de lógica a um caminho tortuoso, impedindoo de aceitar leis lógicas bastante naturais como $1+x=1$ e $x+x=x$. Não obstante, Boole defende que lógica é uma ciência que lida tanto com relação entre classes como com a relação entre proposições. Na minha visão, a separação entre metafísica e lógica defendida por Boole é uma mera ilusão.

Apesar dos grandes feitos do sistema de Boole, tentei mostrar também que o seu sistema lógico tem deficiências. Uma delas já mencionei acima: a não-aceitação de axiomas lógicos naturais. Mas há também o problema das soluções de equações eletivas, que está atrelada à conversão parcial. De acordo com a opinião de Corcoran, com a qual concordo, a solução de equações não é um método de dedução. Argumentei que as fórmulas (1) $x=v y \mathrm{e}$ (2) $x(1-y)$ que seriam supostamente equivalentes não têm o mesmo conjunto de deduções. Portanto, isso coloca em xeque a derivação de (1) a partir de (2), usando-se o método de solução. Como mostrei, a derivação de (2) a partir de (1) faz uso apenas dos axiomas do sistema e dispensa o método de solução. Finalmente, mencionei rapidamente o método de refutação de Boole. 
Em comparação com método de contraexemplos de Aristóteles, o método de Boole é complexo e não-natural.

\section{Referências}

ARISTÓTELES. The Works of Aristotle vol. I. Publicado por William Benton. Chicago: Encyclopaedia Britannica, 1952.

BOOLE, G. George Boole: Selected Manuscript on Logic and its Philosophy. Editado por Ivor Grattan-Guinness e Gérad Bornet. Berlin: Birhäuser Verlag, 1997.

BOOLE, G. The Mathematical Analysis of Logic. England: Thoemmes Press, 1998.

BOOLE, G. The Laws of Thought. New York: Prometheus Books, 2003.

CHATEAUBRIAND, O. Boole on Reference and Universe of Discourse: Reply to John Corcoran. IN: SILVA, J. J. da (Ed.). Logic, Truth and Description: Essays on Chateaubriand's Logical Forms. Campinas, São Paulo: Manuscrito, 2004. p. 173-182.

CORCORAN, J. Boole's Criteria for Validity and Invalidity. Notre Dame Journal of Formal Logic, v. 21, n. 4, p. 609-638, 1980.

CORCORAN, J. Aristotle's Prior Analytics and Boole's Laws of Thought. History and Philosophy of Logic, v. 24, p. 261-288, 2003.

CORCORAN, J. The Principle of Wholistic Reference. IN: SILVA, J. J. da (Ed.). Logic, Truth and Description: Essays on Chateaubriand's Logical Forms. Campinas, São Paulo: Manuscrito, 2004. p. 159-172.

DUMMETT, M. George Boole. IN: DUMMETT, M. (Ed.). Truth and Other Enigmas. London: Duckworth, 1978. p. 66-73.

HEIJENOORT, J. van (Ed.). From Frege to Gödel. Cambridge: Harvard University Press, 1981.

LEWIS, C. I.; LANGFORD, C. H. Symbolic Logic. New York: Dover Publications, 1959.

PEIRCE, C. S. On the algebra of logic. IN: KLOESEL, C. J. W. et. all (eds.) Writings of Charles S. Peirce: a chronological edition, v. 4. Bloomington e Indianapolis: Indiana University Press, 1989, p. 163-209.

QUINE, W. van O. Mathematical Logic. New York: Harper and How, 1940. 